\title{
Economic Incentives and Social Preferences: Causal Evidence of Non-Separability
}

\author{
Marco Faravelli* and Luca Stanca ${ }^{\dagger}$ \\ October 2014 (forthcoming) \\ Journal of Economic Behavior and Organization
}

\begin{abstract}
This paper investigates the hypothesis that the objective function of economic agents is non-separable in economic incentives and social preferences. We study fixed-prize contests in a $2 \times 2$ experimental design, varying orthogonally the degree of competition of the incentive mechanism (all-pay auction vs. lottery) and the presence or absence of social returns to bidding (public good vs. rent seeking). The results indicate that both stronger competition and the presence of the public good have positive main effects on bids. More importantly, we find a negative interaction between stronger competition and the presence of the public good, leading us to reject separability. These findings provide causal evidence that economic incentives may negatively affect pro-social behavior. More generally, they indicate that social preferences should be taken into account for the optimal design of incentive mechanisms.
\end{abstract}

Keywords: Contests; Public goods; Rent-seeking; Social preferences; Separability; Laboratory experiments.

JEL codes: C91; D44; H41.

${ }^{*}$ School of Economics, University of Queensland, Australia. Email: m.faravelli@uq.edu.au

${ }^{\dagger}$ Corresponding author. Department of Economics, University of Milan Bicocca, Italy. Phone: +390264483155. Fax: +3902700439240. E-mail: luca.stanca@unimib.it 


\section{Introduction}

Economists commonly assume that agents' objective functions are separable in economic incentives and social preferences (e.g., Rabin, 1993; Fehr and Schmidt, 1999; Levitt and List, 2007). This implies that responses to economic incentives are independent of individuals' social preferences or, likewise, that pro-social behavior is unaffected by the presence of economic incentives. Several recent theoretical studies, however, suggest that economic incentives may interact with, and often adversely affect, social preferences (e.g. Kreps, 1997; Benabou and Tirole, 2006; Schmidt, 2011). At the empirical level, a growing literature provides evidence for several mechanisms that may explain such an interaction: incentives may provide information about the principal, frame the decision situation, compromise an individual's sense of autonomy, or affect the process by which people learn new preferences (Bowles and Polanía-Reyes, 2012; see also Gneezy et al., 2011).

An important limitation of the recent empirical literature supporting the notion of non-separability is that it only provides indirect evidence. The empirical strategy followed in virtually all the relevant experimental studies is to compare the observed effects of a given incentive mechanism with those that would be predicted theoretically under separability (Bowles and Polanía-Reyes, 2012). ${ }^{1}$ If the performance of the incentive mechanism differs from the theoretical predictions, it is inferred that economic incentives have interacted with social preferences. Such evidence, however, is generally compatible with several alternative explanations other than non-separability, such as the role played by risk attitudes or cognitive demands. Given that an incentive mechanism could under-perform (or over-perform) per se, irrespective of social preferences, the available evidence is generally not informative about the impact of economic incentives on social preferences.

In this paper we argue that, in order to obtain causal evidence of nonseparability between economic incentives and social preferences, it is necessary to compare the effectiveness of incentives while exogenously manipulating the elicitation of social preferences. Causal evidence of non-separability can be obtained only if the performance of economic incentives is systematically related to the elicitation of social preferences. In order to illustrate this point, we focus on prize-based incentive mechanisms for the private provision of public goods as a relevant application.

Several recent studies have investigated the performance of various types of contests as fundraising mechanisms. Most of this literature has focused on either stochastic contests, i.e., lotteries, or deterministic contests, mostly all-pay auctions (e.g. Morgan, 2000; Morgan and Sefton, 2000; Goeree et al., 2005; Landry et al., 2006; Lange et al., 2007; Carpenter et al., 2008;

\footnotetext{
1 "Our empirical strategy (based on experimental results) is to observe the total effect of incentives on behavior and to note whether this differs from the predicted direct effect in order to infer the effects of incentives on (unobserved) social preferences [...]" (Bowles and Polanía-Reyes, 2012, p. 368).
} 
Faravelli and Stanca, 2012), while a smaller number of studies have compared the two types (Davis et al., 2006; Orzen, 2008; Schram and Onderstal, 2009; Corazzini, et al., 2010; Duffy and Matros, 2012; Onderstal et al., 2013) ${ }^{2}$ In a lottery, each player wins the prize with a probability equal to the ratio of her bid and the sum of all bids. On the contrary, in an all-pay auction the highest bidder is awarded the prize with certainty. The two types of contest are characterized by a different marginal effectiveness of effort. The marginal impact of effort is lower in a lottery than in an all-pay auction, where the payoff discontinuity provides a greater incentive to outbid the competitors. As a result, for a finite number of players, revenues are expected to be higher in an all-pay auction than in a lottery. The different marginal impact of effort in the two incentive mechanisms implies that competition is milder in a lottery, while it is stronger in an all-pay auction.

In accordance with the theory, all-pay auctions typically outperform lotteries in the absence of public goods (see, e.g., Davis and Reilly, 1998; Potters et al., 1998; Sheremeta et al., 2012). However, Corazzini et al. (2010) and Orzen (2008) find that this is not the case when these two contests are used as fundraising mechanisms. More specifically, while Corazzini et al. (2010) find a positive and significant difference between the lottery and the allpay auction, Orzen (2008) reports higher bids in the lottery but the difference is not significant. These results suggest an interpretation based on the adverse effect of competition on social preferences. If agents care about social returns to the public good, in addition to their private returns, such other-regarding motive may be crowded out by the competition introduced by the incentive mechanism. While a lottery, representing relatively mild competition, only partially crowds out other-regarding motives, an all-pay auction, characterized by stiffer competition, can be expected to have a stronger crowding out effect.

In the present work we investigate this conjecture by providing a direct test of non-separability between economic incentives and social preferences. To this purpose, we implement a laboratory experiment based on a $2 \times 2$ design by orthogonally manipulating two treatment variables: the degree of competition of the incentive mechanism (all-pay auction vs. lottery) and the presence or absence of social returns (public good vs. rent-seeking). Our key hypothesis is that the degree of competition introduced by the incentive mechanism interacts with agents' attitudes towards the redistribution implied by the public good. More specifically, we hypothesize that a more competitive setting leads agents to be less pro-social - or more anti-social - with respect to redistribution. Importantly, while the adverse effect of stronger competition on attitudes toward redistribution is expected in the public good setting, where bids are shared among group members, such an

\footnotetext{
${ }^{2}$ Konrad (2009) provides a general overview of contest design. See Dechenaux et al. (2014) for a recent comprehensive review of experimental studies on contests.
} 
effect can be ruled out in the rent seeking setting, where bids are not shared among group members. Therefore, non-separability between economic incentives and social preferences can be tested by focusing on the interaction between the all-pay auction mechanism (stronger competition) and the public good setting (presence of redistribution). Under the null hypothesis of separability, the interaction is expected to be positive. A negative interaction between stronger competition and the presence of redistribution would thus provide causal evidence of non-separability.

We should note that, given the payoff externalities that are involved in contests, several other types of social preferences, such as spite or envy, could play a role even in the absence of the public good component (e.g. Herrmann and Orzen, 2008; Eisenkopf and Teyssier, 2013). However, to the extent that these other types of social preferences are orthogonal to the public good component, their presence does not affect the interaction between stronger competition and positive social returns. Sheremeta (2013) suggests various other extensions, besides social preferences, that may interact with the degree of competition, such as mistakes, judgemental biases and utility of winning. As pointed out in Sheremeta (2010), the utility of winning plays a fundamental role in explaining individual bids in contests. This could also explain the negative interaction between competitiveness and pro-social preferences, as the utility of winning may be enhanced in a pure rent-seeking setting, while it may be mitigated in the presence of a public good.

The results indicate that both stronger competition and the presence of the public good have positive (main and simple) effects on bids. Most importantly, we find a significant negative interaction between stronger competition and the presence of the public good. That is, the difference in average bids between the all-pay auction and the lottery is smaller in the presence than in the absence of the public good. This leads us to reject the hypothesis that agents' objective functions are separable in economic incentives and social preferences. Within treatments, average bids are not significantly different from the theoretical predictions in all treatments but the all-pay auction in the public good setting, where we observe substantial and significant underbidding. Under-performance in this treatment is explained by the fact that the share of subjects bidding their entire endowment is lower than predicted.

Two experiments are closely related to ours. The first is a paper by Schram and Onderstal (2009) investigating the performance of alternative auction mechanisms in the presence or absence of the public good component. Their analysis, however, is based on a different setting and does not focus on interactions between incentive mechanisms and social preferences. The second is a recent experiment by Savikhin and Sheremeta (2013) comparing a rent-seeking lottery, a public good game and a third treatment 
in which subjects participate in both games. When the two games are played separately, they observe over-bidding in the rent-seeking game and positive contributions decreasing over time in the public good game. When the games are played jointly, over-bidding in the rent-seeking game is reduced, while public good contributions are not affected by the contest. Thus, they find evidence of a negative interaction between degree of competition and pro-social preferences. However, while we find that increased competition crowds out pro-social preferences, they observe a behavioral spillover from the public good to the contest, with the pro-social preferences exhibited in the public good setting extending to the rent-seeking environment. This is likely to be explained by the different experimental designs. Their subjects play in a pro-social and in a competitive environment, either separately or jointly. As Savikhin and Sheremeta point out, in such a design both strategic uncertainty and path-dependence can explain the direction of the spillover. In our $2 \times 2$ design we embed the public good element in a contest while independently manipulating the degree of competition of the contest.

The paper is structured as follows. Section 2 outlines the theoretical framework. Section 3 describes the experiment. Section 4 presents the results. Section 5 provides a discussion of the main findings, while Section 6 concludes. Details on the theoretical predictions and experimental instructions are provided in Appendix.

\section{Theory}

Consider a set $N=\{1, \ldots, n\}$ of two or more agents who take part in an all-pay contest. All agents are endowed with the same budget $\omega$ and compete for a prize $\Pi$ they all equally value. Differently from a standard contest, each agent's bid is multiplied by $\alpha \in[0, n)$ and shared equally among all agents. Hence, when $\alpha=0$, we are considering a pure rent-seeking contest (RS), while when $\alpha>0$ we are in a public good setting (PG).

The expected payoff for the generic agent $i$ who bids $x_{i}$ is given by

$$
\omega-x_{i}+\frac{\alpha}{n} \sum_{j \in N} x_{j}+p\left(x_{i}, \mathbf{x}_{-i}\right) \Pi
$$

where $\mathbf{x}_{-i}$ is the vector of bids of all players except $i$, while $p\left(x_{i}, \mathbf{x}_{-i}\right)$ represents $i$ 's probability of winning the prize.

We consider two types of contest, a lottery (LOT) and an all-pay auction (APA), that differ in the way the prize is awarded. In LOT, a player wins the prize with a probability equal to the ratio between her bid and the sum of all bids: 


$$
p^{L O T}\left(x_{i}, \mathbf{x}_{-i}\right)=\left\{\begin{array}{c}
\frac{x_{i}}{\sum_{j \in N} x_{j}} \text { if } \sum_{j \in N} x_{j}>0 \\
\frac{1}{n} \text { otherwise }
\end{array} .\right.
$$

In APA, the highest bid is awarded the prize, and ties are randomly broken:

$$
p^{A P A}\left(x_{i}, \mathbf{x}_{-i}\right)=\left\{\begin{array}{c}
\frac{1}{\#\left\{j \in N: j \in \arg \max _{l \in N} x_{l}\right\}} \text { if } i \in \arg \max _{j \in N} x_{j} \\
0 \text { otherwise }
\end{array} .\right.
$$

As it is well known, the probability of winning the prize in both APA and LOT can be described by a Tullock contest success function (see Tullock, 1980)

$$
p\left(x_{i}, \mathbf{x}_{-i}, \rho\right)=\frac{x_{i}^{\rho}}{\sum_{j \in N} x_{j}^{\rho}},
$$

where the parameter $\rho$ describes the degree of competition in the contest (the higher $\rho$, the more competitive the contest), with $p\left(x_{i}, \mathbf{x}_{-i}, 1\right)=p^{L O T}$ and $p\left(x_{i}, \mathbf{x}_{-i}, \infty\right)=p^{A P A}$, respectively. ${ }^{3}$ Player $i$ 's utility can therefore be written as

$$
U_{i}=\omega-x_{i}+\frac{\alpha}{n} \sum_{j \in N} x_{j}+\frac{x_{i}^{\rho}}{\sum_{j \in N} x_{j}^{\rho}} \Pi .
$$

As shown in the following proposition, in LOT there exists a unique Nash equilibrium in pure strategies. Provided that the endowment is not too small, total bids are $(n-1) \frac{\Pi}{n-\alpha} \cdot 4$

Proposition 1 If $\omega \leq \frac{n-1}{n} \frac{\Pi}{n-\alpha}$ LOT has a unique Nash equilibrium in which everyone bids the whole endowment, with total bids equal to nw.

If $\omega>\frac{n-1}{n} \frac{\Pi}{n-\alpha}$ LOT has a unique Nash equilibrium in which every player bids $x^{L O T}=\frac{n-1}{n} \frac{\Pi}{n-\alpha}$, with total bids equal to $(n-1) \frac{\Pi}{n-\alpha}$.

The next proposition outlines the equilibrium of APA for any value of $\omega$. We focus on symmetric equilibria, while asymmetric equilibria are discussed in Section 5..$^{5}$ As in the case of LOT, when $\omega$ is below a critical value, APA has a unique symmetric equilibrium in which every player bids the whole endowment. Beyond this critical value, we distinguish between an intermediate and a large endowment range. For both ranges of endowment,

\footnotetext{
${ }^{3}$ Define $p\left(x_{i}, \mathbf{x}_{-i}, \infty\right)=\lim _{\rho \rightarrow \infty} \frac{x_{i}^{\rho}}{\sum_{j \in N} x_{j}^{\rho}}$.

${ }^{4}$ This proposition presents a result already outlined in other papers, such as Morgan (2000), Orzen (2008) and Duffy and Matros (2012). However, we state it formally, and provide the proof in the appendix, for completeness.

${ }^{5}$ As for the case of Proposition 1, Proposition 2 reports results already present in previous papers (see Orzen, 2008; Duffy and Matros, 2012). However, its proof will help us analyze the asymmetric equilibria.
} 
Table 1: Expected total bids as a function of $\rho$ and $\alpha$

\begin{tabular}{lcc}
\hline \hline & $\operatorname{RS}(\alpha=0)$ & PG $(\alpha>0)$ \\
\hline LOT $(\rho=1)$ & $\frac{n-1}{n} \Pi$ & $\frac{n-1}{n} \frac{n}{n-\alpha} \Pi$ \\
APA $(\rho \rightarrow \infty)$ & $\Pi$ & $\frac{n}{n-\alpha} \Pi$ \\
\hline
\end{tabular}

Note: See Propositions (1) and (2) for details.

there exists a unique symmetric equilibrium with total expected bids equal to $\frac{n}{n-\alpha} \Pi .^{6}$

Proposition 2 If $\omega \leq \frac{\Pi}{n-\alpha}$ APA has a unique symmetric Nash equilibrium in which everyone bids $\omega$, with total bids equal to $n \omega$.

If $\frac{\Pi}{n-\alpha}<\omega<\frac{n}{n-\alpha} \Pi$ APA has a unique symmetric Nash equilibrium such that each player bids $\omega$ with probability $q \in(0,1)$ and with probability $1-q$ randomizes according to $F(x)=\left(\frac{n-\alpha}{n} \frac{x}{\Pi}\right)^{\frac{1}{n-1}}$ on the support $[0, \bar{x}]$. $q$ is the unique solution to following equation

$$
\frac{1-(1-q)^{n}}{q}=\frac{n-\alpha}{\Pi} \omega,
$$

while $\bar{x}$ is given by the following

$$
\bar{x}=(1-q)^{n-1} \frac{n}{n-\alpha} \Pi .
$$

Total expected bids amount to $\frac{n}{n-\alpha} \Pi$.

If $\omega \geq \frac{n}{n-\alpha} \Pi$ APA has a unique symmetric Nash equilibrium such that each player randomizes according to $F(x)=\left(\frac{n-\alpha}{n} \frac{x}{\Pi}\right)^{\frac{1}{n-1}}$ on the support $\left[0, \frac{n}{n-\alpha} \Pi\right]$. Total expected bids amount to $\frac{n}{n-\alpha} \Pi$.

Table 1 reports expected total bids, as a function of $\rho$ and $\alpha$, under the assumption that agents maximize (2) and that budget constraints are nonbinding. ${ }^{7} \quad$ As it can be observed, total expected bids are higher in APA than in LOT irrespective of $\alpha$. Likewise, they are higher in PG than in RS irrespective of $\rho$. In addition, there is a positive interaction between $\rho$ and $\alpha$. That is, the difference between APA and LOT is larger when bids are contributions to a public good $(\alpha>0)$ than in a pure rent-seeking contest $(\alpha=0)$.

Consider now an alternative objective function that explicitly accounts for social preferences through an additional other-regarding component:

\footnotetext{
${ }^{6}$ The large endowment case is equivalent to an all-pay auction in which bidders are not budget constrained. See Baye et al. (1996) for a full equilibrium characterization.

${ }^{7}$ Throughout the paper, by non-binding constraint we refer to the case in which expected equilibrium bids are lower than the budget. In the case of APA this includes both the intermediate and large endowment ranges.
} 


$$
V_{i}=U_{i}+f(\rho) \frac{(n-1)}{n} \alpha \sum_{j \in N} x_{j} .
$$

The term $\frac{(n-1)}{n} \alpha \sum_{j \in N} x_{j}$ represents the external returns to the public good, i.e., the total earnings of other group-members, and $f(\rho)$ is their weight in the objective function. This weight can be either positive, in the case of pro-social preferences, or negative in the case of anti-social preferences. Our key assumption is that the weight of external returns is a function of $\rho$ : that is, economic incentives and social preferences are non-separable. More specifically, we assume that $\frac{d f}{d \rho}<0$ : the higher the degree of competition of the incentive mechanism, the lower the weight of external returns in the objective function. This means that, when the environment is more competitive, an individual obtains a smaller benefit, or a larger cost, from others' payoffs. Note that, in the absence of the public good component $(\alpha=0)$, expression (3) reduces to (2), i.e., agents behave as selfish payoff maximizers. On the other hand, when $\alpha>0$, stronger competition either crowds out pro-social preferences, when $f(\rho)>0$, or exacerbates anti-social preferences, when $f(\rho)<0 .{ }^{8}$

Suppose $\alpha>0$ and let us restrict the attention to the case of non-binding budget constraints. Focusing on the other-regarding component in equation (3), for a given $\alpha$ the marginal benefit of external returns is higher in LOT $(\rho=1)$ than in APA $(\rho \rightarrow \infty)$. Non-separability thus reduces the returns to bidding in APA, relative to LOT, when $\alpha>0$, while it has no effects when $\alpha=0$. As a consequence, while a positive interaction between $\rho$ and $\alpha$ is expected under the null hypothesis of separability, a negative interaction provides evidence of the adverse effect of competition on social preferences. This negative interaction, arising from the non-separability of competition and social preferences, is the key prediction we wish to test in our experiment.

\section{The experiment}

This section presents the experiment. We start by describing the experimental design, the task and the treatments. We then provide details on the procedures. Finally, we illustrate the hypotheses to be tested.

\subsection{Design}

Our experiment is based on a $2 \times 2$ factorial design, as illustrated in Table 2. The treatment variables are the degree of competition of the incentive

\footnotetext{
${ }^{8}$ Note that the predictions would be qualitatively unchanged if agents were assumed to be impure altruists who obtain utility from contributing to the public good, i.e. $V_{i}=$ $U_{i}+f(\rho) x_{i}$ when $\alpha>0$, as long as $\frac{d f}{d \rho}<0$.
} 
Table 2: Experimental design

\begin{tabular}{lcc}
\hline \hline & RS $(\alpha=0)$ & PG $(\alpha>0)$ \\
\hline LOT $(\rho=1)$ & T1 & T2 \\
APA $(\rho \rightarrow \infty)$ & T3 & T4 \\
\hline
\end{tabular}

Note: treatments implemented within subjects with a cross-over design, as detailed in

Table 3.

mechanism ( $\rho=1$ in LOT vs. $\rho \rightarrow \infty$ in APA) and the presence or absence of the public good component ( $\alpha=0$ in RS vs. $\alpha>0$ in PG).

The experimental task is as follows. In each round subjects are endowed with a given amount in euros and are randomly matched in groups of four. They can bid any integer amount in euro cents, between 0 and their endowment, to win a fixed prize in euros. The winner in the group of four subjects, and total earnings, are determined according to the treatment (incentive mechanism-environment pair) in place for that round.

We implement the following four treatments:

T1 LOT-RS: Subjects receive a lottery ticket for each euro cent bid. The winner is chosen randomly, and each bidder's probability of winning is equal to the ratio of their bid to the total amount of bids by all members of their group in that round.

T2 LOT-PG: The winner is chosen as in (1). In addition, the total amount of bids is multiplied by 1.5 and shared equally among the four group members.

T3 APA-RS: The winner is the player who bids the highest amount. In the event of a tie, the winning bidder is randomly chosen among those who bid the highest amount.

T4 APA-PG: The winner is chosen as in (3). In addition, the total amount of bids is multiplied by 1.5 and shared equally among the four group members.

The endowment and the prize are kept fixed across treatments: $\omega=8$ euros and $\Pi=16$ euros in all four treatments. We chose to set $\Pi=2 \omega$ in order to make the incentive salient, while at the same time ensuring that in equilibrium the budget constraint is not binding in all four treatments.

\subsection{Procedures}

We run four sessions, with 24 subjects participating in each session, for a total of 96 subjects. Treatments were implemented in a within-subjects design. Each treatment was run over 10 rounds, for a total of 40 rounds (4 phases of 10 rounds each) in each session. In order to minimize the consequences 
Table 3: Details of cross-over design

\begin{tabular}{lcccc}
\hline \hline & Phase 1 & Phase 2 & Phase 3 & Phase 4 \\
\hline Session 1 & T1 & T2 & T3 & T4 \\
Session 2 & T2 & T1 & T4 & T3 \\
Session 3 & T3 & T4 & T1 & T2 \\
Session 4 & T4 & T3 & T2 & T1 \\
Note: each treatment was implemented within subjects for 10 rounds, for a total of 40 \\
rounds overall (four phases of 10 rounds each).
\end{tabular}

of possible order effects, we implemented a cross-over design by reversing the order of the treatments in the four ten-round phases of each session, as detailed in Table 3.

In order to avoid repeated game effects, subjects were informed that groups would be randomly formed in each round. Unknown to subjects, groups of four subjects were formed in each round by randomly drawing from fixed sets of 8 subjects. We thus obtain three independent matchinggroup observations per session, for a total of 12 independent group-level observations.

In each session, subjects were randomly assigned to a computer terminal at their arrival. To ensure public knowledge, instructions were distributed and read aloud (see Appendix B for the instructions, translated from Italian). In order to ensure understanding of the experimental task, sample questions were distributed before the start of each phase. The answers were privately checked and, if necessary, individually explained to the subjects. At the end of each round, subjects were informed about the bids and payoffs of each group member. At the end of the last round, subjects were informed of their total payoff in euros, based on one round randomly drawn out of 40 . Subjects were asked to answer a short questionnaire about socio-demographic background, and were then paid in private.

The experiment took place at the Experimental Economics Laboratory of the University of Milan Bicocca in December 2012. Subjects were students from the undergraduate population of the University recruited by public announcement. Subjects earned on average 12.25 euros for sessions lasting between 70 and 80 minutes. The experiment was computerized using the z-Tree software (Fischbacher, 2007).

\subsection{Hypotheses}

Table 4 summarizes expected individual bids in euro cents for each of the four treatments, under the null hypothesis that subjects maximize (2). Given our parameter calibration, in equilibrium individual bids are 300 and 480 euro cents in LOT-RS and LOT-PG, respectively (Proposition 1). Individual expected bids are 400 and 640 in APA-RS and APA-PG, respectively 
Table 4: Expected individual bids

\begin{tabular}{lcc}
\hline \hline & $\mathrm{RS}(\alpha=0)$ & $\mathrm{PG}(\alpha=1.5)$ \\
\hline LOT $(\rho=1)$ & 300 & 480 \\
APA $(\rho \rightarrow \infty)$ & 400 & 640
\end{tabular}

Note: See Propositions (1) and (2) for details. Expected individual bids are expressed in euro cents.

(Proposition 2). ${ }^{9}$ Standard theory thus predicts positive simple effects of APA relative to LOT (in both RS and PG) and positive simple effects of PG relative to RS (in both LOT and APA). In addition, a positive interaction is predicted between APA and PG. That is, the absolute difference between APA and LOT is expected to be larger in PG than in RS.

The predictions above are derived in the absence of social preferences, as described in (2). In the presence of social preferences, under the null of separability the size of the main effects would be affected but the prediction of a positive interaction between APA and PG would be unaffected. On the other hand, under non-separability between incentives and social preferences, as expressed in (3), the difference between the marginal benefit of bidding in APA and LOT would become smaller in the public good environment $(\alpha>$ $0)$, while it would be unaffected in the rent-seeking environment $(\alpha=0)$. Therefore, a negative interaction between APA and PG provides evidence of non-separability.

\section{Results}

This section presents the experimental results. We start by reporting test results for treatment effects. Next, we compare actual and predicted bids within treatments and examine the effects of repetition. Finally, we consider bidding behavior at individual level.

\subsection{Treatment effects}

Figure 1 compares observed and predicted individual bids in the four treatments. Observed average individual bids are 299 and 451 euro cents in LOTRS and LOT-PG, respectively, while 380 and 494 euro cents, respectively, in APA-RS and APA-PG. The experimental data clearly indicate positive main effects for both APA and PG. However, in contrast with the null hypothesis of separability, a negative interaction is observed between APA and PG.

\footnotetext{
${ }^{9}$ More specifically, in APA-RS subjects are predicted to randomize between 0 and 257 according to $F(x)=0.085 x^{\frac{1}{3}}$ and bid 800 with probability 0.46 , with an expected bid equal to 400; in APA-PG, subjects are predicted to randomize between 0 and 21 according to $F(x)=0.073 x^{\frac{1}{3}}$ and bid 800 with probability 0.8 , resulting in an expected bid equal to 640 .
} 
Figure 1: Main treatment effects and interactions (euro cents)

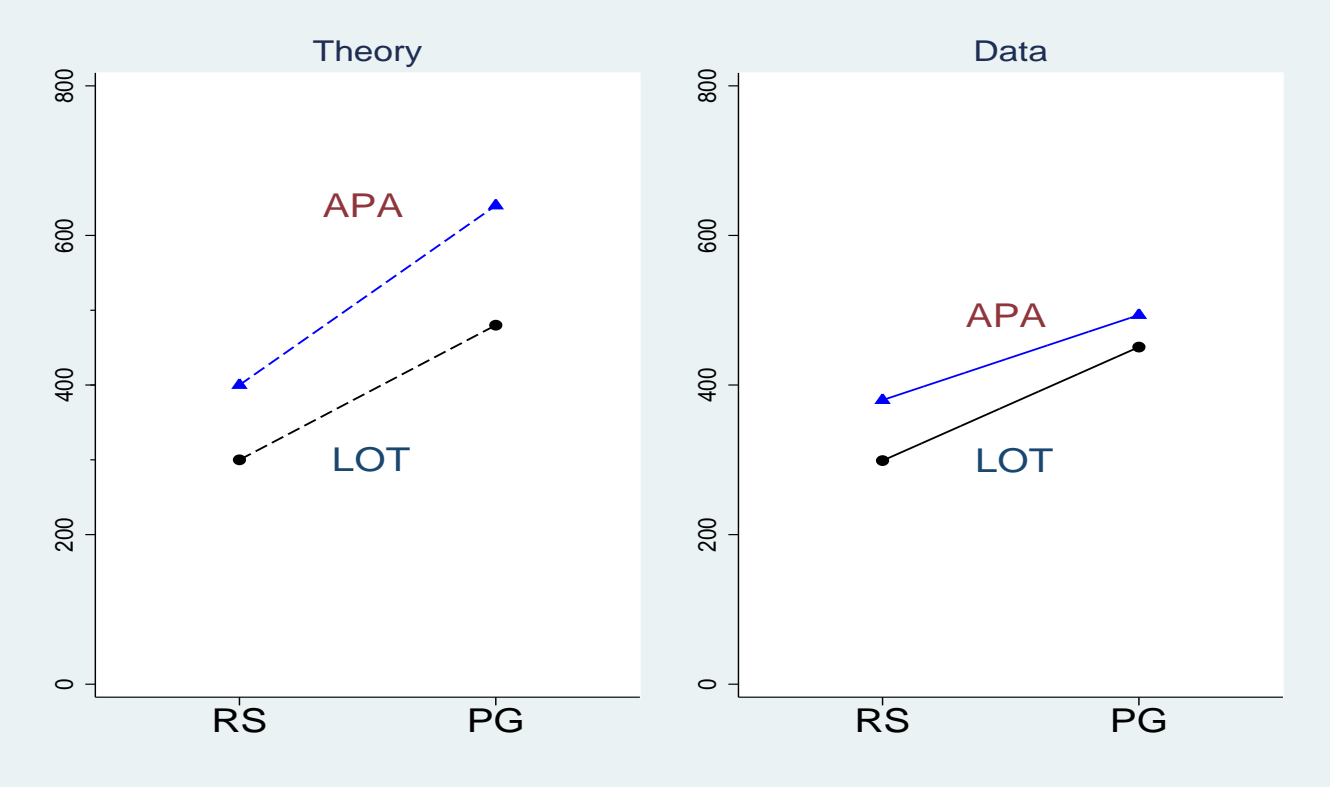

That is, the difference between APA and LOT is smaller in the presence of the public good than in the rent-seeking environment.

In order to test the significance of treatment effects, we use a regression model that explains observed average group-level bids $(\bar{x})$ with dummy variables for treatment effects (APA and PG) and their interaction. The model includes dummy variables for the order of treatments within sessions and, to account for learning effects, dummy variables for the second half of the session and the second half of each phase. The resulting specification is as follows:

$$
\begin{aligned}
\bar{x}_{i t}= & \beta_{0}+\beta_{1} P G_{i t}+\beta_{2} A P A_{i t}+\beta_{3}\left(P G_{i t} * A P A_{i t}\right)+ \\
& +\sum_{j=1}^{2} \gamma_{j} O R D_{j i}+\sum_{j=1}^{2} \delta_{j} E X P_{j t}+\varepsilon_{i t}
\end{aligned}
$$

where $i=1, \ldots, 24$ indicates a group of four subjects, $t=1, \ldots, 40$ denotes the round within the session, $O R D$ includes a dummy for sessions starting with $A P A$ (sessions 3 and 4) and a dummy for sessions starting with $P G$ (sessions 2 and 4), EXP includes a dummy for the second half of the session (EXP (EXP , rounds 6-10,16-20, 26-30, or 36-40). Since the effect of truncation is negligible, as less than $3 \%$ of the group-level average bids are 0 or 800 , the model is estimated by OLS. Standard errors are clustered by independent matching groups of 8 individuals.

Table 5 presents estimation results for treatment effects. Column (1) reports the main effects of $\mathrm{APA}$ and PG, i.e., the average treatment effects 
Table 5: Main treatment effects and interaction

\begin{tabular}{|c|c|c|c|}
\hline & $\begin{array}{c}(1) \\
\text { Main effects }\end{array}$ & $\begin{array}{c}(2) \\
\text { Simple effects }\end{array}$ & $\begin{array}{c}(3) \\
\text { Simple effects }\end{array}$ \\
\hline APA & $\begin{array}{c}61.9^{* * *} \\
(10.4)\end{array}$ & $\begin{array}{c}80.9^{* * *} \\
(14.4)\end{array}$ & $\begin{array}{c}42.8^{* *} \\
(14.9)\end{array}$ \\
\hline PG & $\begin{array}{c}132.8^{* * *} \\
(15.9)\end{array}$ & $\begin{array}{c}151.8^{* * *} \\
(18.4)\end{array}$ & $\begin{array}{c}113.7^{* * *} \\
(19.6)\end{array}$ \\
\hline $\mathrm{APA} * \mathrm{PG}$ & & $\begin{array}{c}-38.1^{* *} \\
(20.6)\end{array}$ & \\
\hline $\mathrm{LOT}^{*} \mathrm{RS}$ & & & $\begin{array}{c}-38.1^{* *} \\
(20.6)\end{array}$ \\
\hline$R^{2}$ & 0.21 & 0.22 & 0.22 \\
\hline Observations & 960 & 960 & 960 \\
\hline \multicolumn{4}{|c|}{$\begin{array}{l}\text { Note: Dependent variable: average group-level bids in euro cents. The figures reported } \\
\text { are OLS estimates of treatment effects, in euro cents, relative to the relevant omitted } \\
\text { treatment(s). Standard errors (in brackets) clustered by independent matching group. } \\
\text { Columns }(2) \text { and }(3) \text { report the simple effects of APA in RS and PG, respectively, and } \\
\text { the simple effects of PG in LOT and APA, respectively. Estimates of the interaction } \\
\text { term in columns }(2) \text { and }(3) \text { are identical by construction. All specifications include } \\
\text { dummy variables for treatment order and experience. }{ }^{*} \text { denotes significance at } 0.10 \text { leve } \\
(* * \text { at } 0.05, * * * \text { at } 0.01) \text { for the relevant hypothesis. }\end{array}$} \\
\hline
\end{tabular}

assuming no interaction. The estimates indicate that both APA and PG have a positive and significant effect on bids on average. The main effects are also quantitatively relevant (61.9 and 132.8 euro cents, respectively), when compared with the size of the endowment (8 euros). Columns (2) and (3) report the simple effects of APA and PG and their interaction. The estimates indicate that the simple effects of APA are 80.9 and 42.8 euro cents in RS and PG, respectively, while the simple effects of $\mathrm{PG}$ are 151.8 and 113.7 euro cents in LOT and APA, respectively. All four simple effects are strongly statistically significant.

Result 1: Both stronger competition and the presence of the public good have positive and significant effects on bids.

More importantly, the results indicate that there is a negative, rather than positive, interaction between APA and PG. This negative interaction (-38.1 euro cents) is not only statistically significant ( $p=0.04$ for the relevant onesided hypothesis) but also quantitatively relevant (about 5 per cent of agents' endowment). ${ }^{10}$ This finding provides causal evidence of non-separability.

Result 2: The separability between economic incentives and social preferences is rejected by the data.

\footnotetext{
${ }^{10}$ Note that the estimates of the interaction term in columns (2) and (3) are identical by construction.
} 
Figure 2: Average individual bids over rounds, by treatment
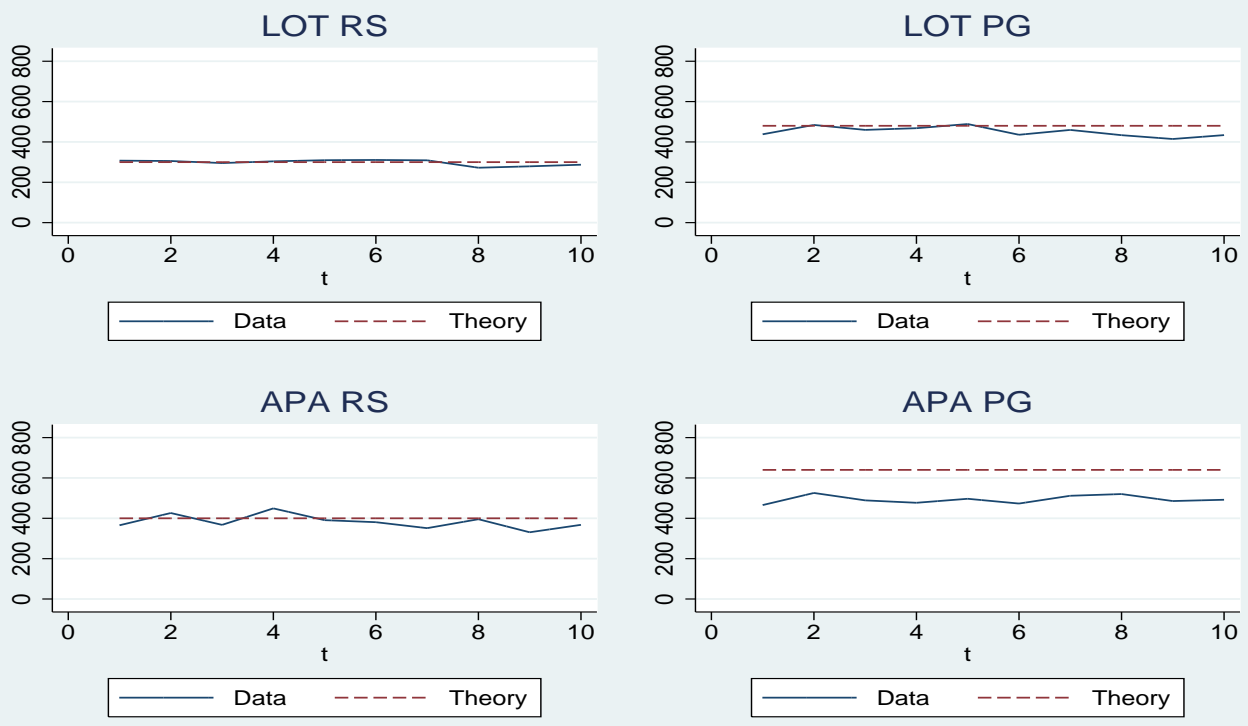

The dummy variables for order effects, not reported in Table 5, indicate that bids are significantly lower, on average, in sessions (3 and 4) where APA is played in the first 20 rounds $\left(O R D_{1}=-85.9\right.$, $p=0.03)$, while there are no significant differences for sessions (2 and 4) where $\mathbf{P G}$ is played in the first 20 rounds $\left(O R D_{2}=7.3\right.$, $p=0.83)$. The dummy variables for experience effects are negative but not significant both across phases $\left(E X P_{1}=-12.6, p=0.25\right)$ and within phases $\left(E X P_{2}=-15.4, p=0.06\right)$. It should be noted that, given the cross-over design we adopted, as described in Table 3, order and experience effects are by construction orthogonal to the treatment effects.

\subsection{Observed vs. predicted bids within treatments}

Figure 2 compares observed and predicted average bids by treatment over 10 rounds, averaging across different subjects and sessions. Two features of the experimental data are evident. First, there is only a slight decline of average bids over successive rounds within phases, without substantial differences in the patterns observed in the four treatments. Second, average bids are remarkably close to predictions in both LOT treatments and in the APARS treatment. On the other hand, the deviation from the expected bid is substantial in APA-PG.

Table 6 presents tests of the significance of the difference between observed and predicted average group bids, for each treatment, based on the specification in (5). The difference in euro cents between observed and predicted bids is -1.1 in LOT-RS, -29.3 in LOT-PG, and -29.1 in APA-RS, and 
Table 6: Significance of deviations from prediction, by treatment

\begin{tabular}{lcccc}
\hline \hline & LOT-RS & LOT-PG & APA-RS & APA-PG \\
\hline Observed - Predicted & -1.1 & -29.3 & -20.1 & $-146.4^{* * *}$ \\
& $(25.6)$ & $(27.9)$ & $(19.9)$ & $(22.2)$ \\
\hline Observations & 240 & 240 & 240 & 240 \\
\hline Note: Dependent variable: deviation of average group bid from prediction, in euro cents. \\
OLS estimates, standard errors (in brackets) clustered by independent matching group. \\
$*$ denotes significance at 0.10 level (** at $0.05,{ }^{* * *}$ at 0.01 ) for the relevant hypothesis.
\end{tabular}

none of these differences is statistically significant. In APA-PG the deviation from the expected bid is instead large (-146.4) and strongly significant $(p=0.00)$.

Result 3: Within treatments, average bids are not significantly different form predictions in both LOT treatments and in APARS. On the other hand, there is significant under-bidding in APAPG.

It should be observed that, while we find average bids that are generally consistent with the theory, and under-bidding only in APA-PG, over-bidding is generally observed in pure rent-seeking contests (see Dechenaux et al., 2014). ${ }^{11}$ In an overview of the experimental literature on contests, Sheremeta (2013) provides different explanations for over-bidding, such as utility of winning, otherregarding preferences and the relative size of the endowment. The latter is likely to explain the difference in overall bidding behavior between our findings and existing studies, since in our experiment agents' endowment is substantially smaller than the prize (800 vs 1600). ${ }^{12}$ As shown in Price and Sheremeta (2011) and Sheremeta (2011), overbidding is positively related to the size of the endowment (relative to the prize). The reason is that, if people make mistakes when bidding, they are generally more likely to over-bid when their endowment is relatively large (see Sheremeta, 2013).

\subsection{Effects of repetition}

Figure 3 displays average bids by treatment over 40 rounds (four phases of 10 rounds). Bids display a downward trend over rounds in both LOT treatments. The difference in euro cents between the two halves of the session is -90.7 in LOT-RS $(p=0.06)$ and -106.3 in LOT-PG $(p=0.04)$, respectively.

\footnotetext{
${ }^{11}$ When contests are used to finance public goods, Orzen (2008) and Corazzini et al. (2010) find over-bidding in the lottery, while average bids are consistent with the predictions in the all-pay auction.

${ }^{12}$ We thank an anonymous referee for pointing this out.
} 
Figure 3: Average contributions, over rounds
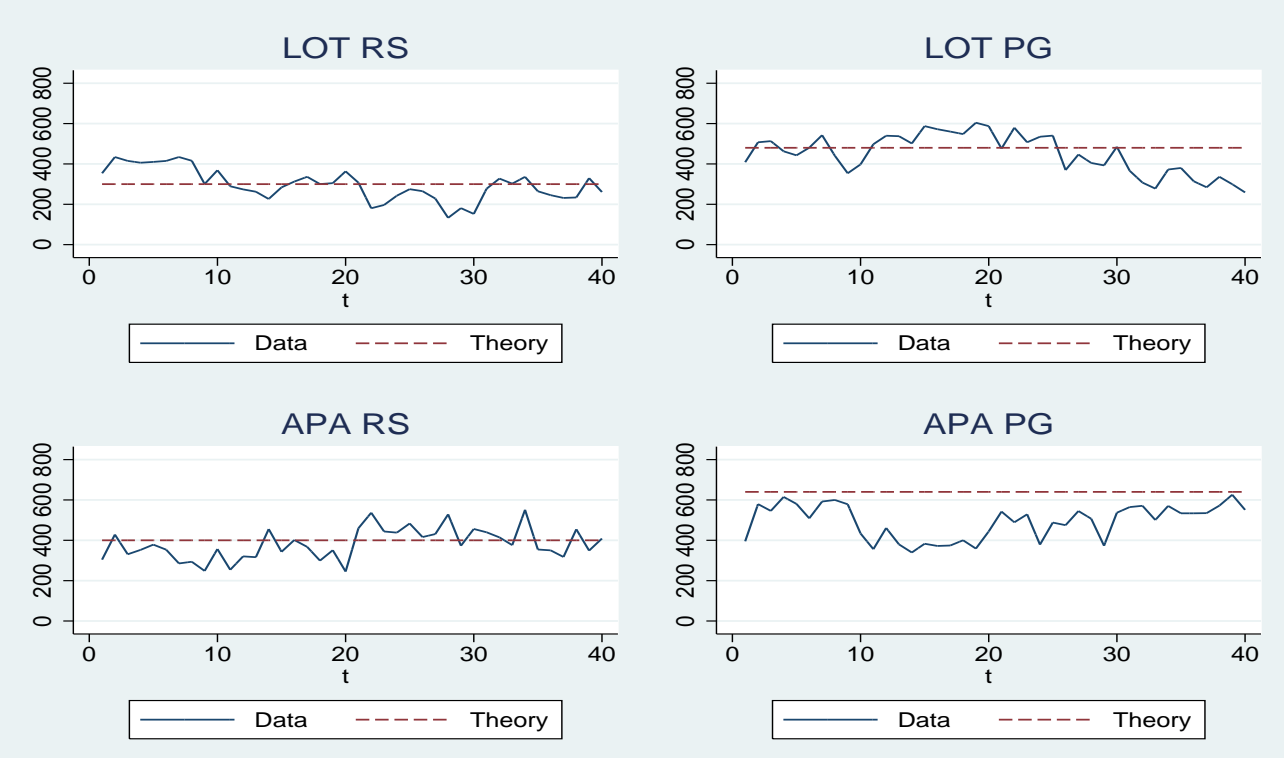

The opposite pattern is observed, instead, in both APA treatments: the difference between the two halves of the session is 85.3 in APA-RS $(p=0.02)$ and 61.3 in APA-PG $(p=0.16)$, respectively. Averaging across RS and PG, the difference is -98.5 for LOT $(p=0.03)$ and 73.3 for APA $(p=0.03)$.

Result 4: Repetition has a negative (positive) effect on bids in LOT (APA) treatments.

What does experience imply for the treatment effects presented above? Table 7 compares treatment effects and treatment interactions between rounds 1-20 and rounds 21-40. Experience increases the main effect of APA, which is negative (-7.1 euro cents) and not significant in periods 1-20, while large (168.9 euro cents) and strongly significant in periods 21-40. On the other hand, experience has a negative and relatively small impact on the main effect of PG (159.6 and 144 euro cents in rounds 1-20 and 21-40, respectively). More importantly, the negative interaction between APA and PG is larger (-42.3 euro cents) and significant in the second half of the session. This is an interesting result, as it indicates that the evidence of non-separability is not eliminated, but rather reinforced, by experience.

\subsection{Individual behavior}

Figure 4 compares the cumulative distribution of individual bids across treatments. Individual behavior is strikingly different depending on the incentive mechanism implemented. In LOT treatments, although there exists a symmetric pure strategy equilibrium, bids are almost uniformly distributed between 0 and 800. In APA treatments, consistently with the theory, bidding 
Table 7: Treatment effects and interaction, by rounds

\begin{tabular}{lcc}
\hline \hline & Rounds 1-20 & Rounds 21-40 \\
\hline APA & -7.1 & $168.9^{* * *}$ \\
& $(41.4)$ & $(32.6)$ \\
PG & $159.6^{* * *}$ & $144.0^{* * *}$ \\
& $(26.9)$ & $(24.6)$ \\
APA ${ }^{*}$ PG & -33.8 & $-42.3^{*}$ \\
& $(44.6)$ & $(28.8)$ \\
\hline$R^{2}$ & 0.17 & 0.27 \\
Observations & 480 & 480 \\
\hline
\end{tabular}

Note: Dependent variable: average group-level bid in euro cents. OLS estimates,

standard errors (in brackets) clustered by independent matching group. All specifications include dummy variables for treatment order and experience. ${ }^{*}$ denotes significance at 0.10 level $\left(* *\right.$ at $0.05,{ }^{* * *}$ at 0.01$)$ for the relevant hypothesis.

is almost bimodal. Interestingly, the within-subject share of overall bid variability is 59 per cent in LOT treatments and 71 per cent in APA treatments. This difference is consistent with the different nature of the equilibria in LOT (pure strategy) and APA (mixed strategy).

Individual bidding behavior also allows us to better understand the reasons for aggregate under-performance of APA in the PG environment. In APA-RS the observed fraction of bids equal to the entire endowment (41 per cent) is relatively close to the theoretical prediction (46 per cent). In APA$\mathrm{PG}$, instead, while the theoretical prediction is 80 per cent, only 55 per cent of bids are equal to the endowment. The under-performance of APA in the PG setting is thus largely explained by the fact that the share of individual bids equal to the entire endowment is lower than predicted.

\section{Discussion}

Our key finding is the negative interaction between the all-pay auction mechanism, characterized by stronger competition, and the public good environment, characterized by the presence of positive social returns to bidding. This leads us to reject the hypothesis of separability between economic incentives and social preferences. The strength of our experimental design is that it allows us to rule out any interpretations of the findings that can explain why the all-pay auction under-performs relative to the lottery, but not why such under-performance is stronger in the presence than in the absence of the public good.

Consider, for example, an interpretation based on subjects' familiarity with the two types of contests. While experimental subjects are generally well acquainted with lotteries, they may have never experienced an all-pay 
Figure 4: Distribution of individual bids, by treatment
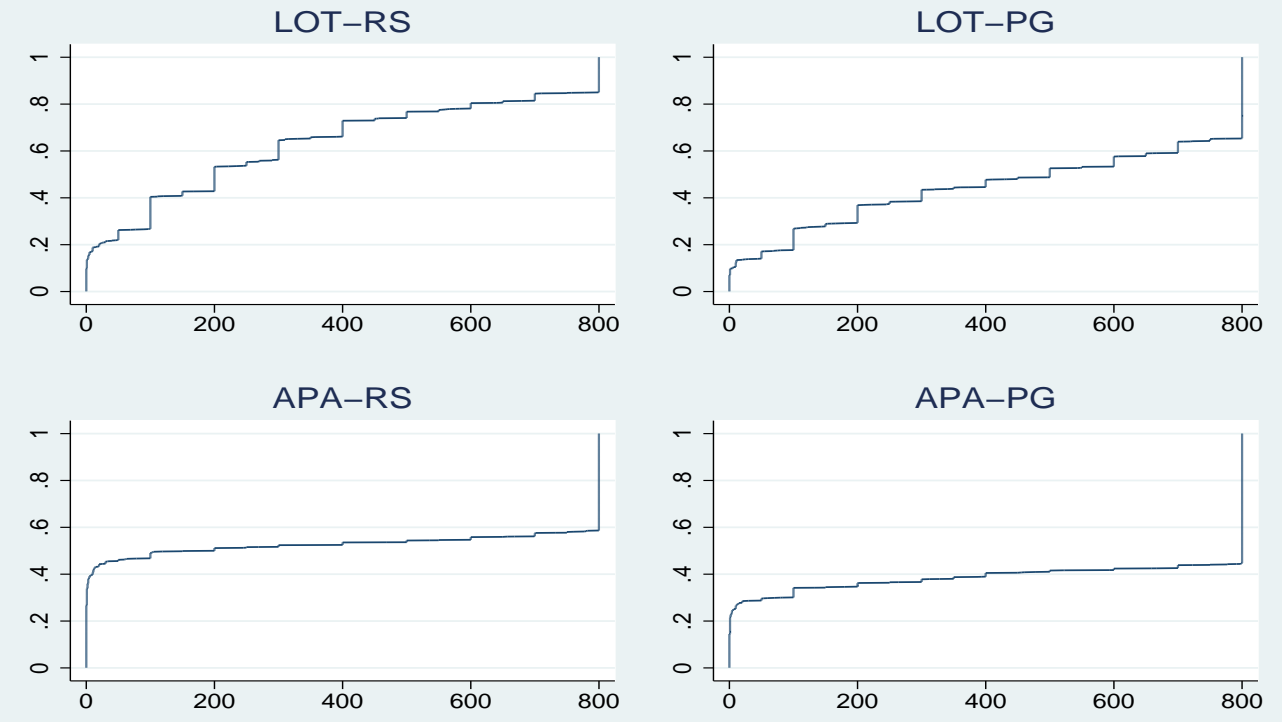

auction. Because of this lack of experience, subjects may have a propensity to abstain from bidding, or bid more cautiously, in APA. However, while nonfamiliarity with the all-pay auction is consistent with the under-performance of APA relative to LOT in the presence of the public good, it is at odds with the fact that there is no such under-performance in the absence of the public good, where average bids are very close to the theoretical predictions.

The question is therefore whether there are explanations of the negative interaction other than the effect of competition on social preferences. One simple explanation would be that the predicted individual bid in the APAPG treatment is substantially higher than in the other treatments (see Table 4) and also relatively close $(80 \%)$ to the value of the endowment. Therefore, under-bidding in APA-PG could be due to the specific calibration of the experiment. This interpretation, however, is unlikely to be valid for two main reasons. First, our test for non-separability is rather conservative, as it requires that the APA-PG interaction is not only negative, but also sufficiently strong to override the positive interaction that would be observed under the null (Figure 1). Second, the predictions for the APA treatments are expected bids of (symmetric) mixed strategy equilibria. Subjects are predicted to randomize between their full endowment, with a given probability, and a given support around zero otherwise. Empirically, as shown in Figure 4, the distribution of individual bids is indeed qualitatively consistent with the predictions, both in APA-RS and APA-PG. The under-performance of APA-PG relative to APA-RS reflects the fact that the fraction of individuals bidding their entire endowment is higher than predicted in the absence of the public good ( $50 \%$ vs. $46 \%$ ), while it is lower than predicted in the presence of the public good (66\% vs. $80 \%$ ). 
An alternative explanation could be based on the high cognitive costs of the all-pay auction relative to the lottery. An experimental subject may have never come across an all-pay auction. In addition, she may find this mechanism more difficult, both cognitively and strategically, than a lottery. In this perspective, the difference in the cognitive demands of APA and LOT might be exacerbated by the presence of the public good component. The interaction between cognitive demands and the presence of an additional redistribution mechanism could therefore explain the lower participation observed to a substantial extent in APA-PG, while only to a smaller extent in APA-RS. Such an explanation, however, is at odds with the effects of repetition. Under the cognitive cost interpretation of the negative APAPG interaction, cognitive demands should become less relevant as subjects become more experienced. We find, instead, that the negative interaction between the all-pay auction mechanism and the public good environment is reinforced by repetition (Table 7 ).

A more fundamental issue concerning the interpretation of the experimental findings is that the theoretical analysis in Section 2 only focuses on symmetric equilibria. Therefore, there may exist asymmetric equilibria for the all-pay auction that are consistent with the findings, without doing away with separability. In Appendix A we characterize the asymmetric equilibria of APA for any prize-endowment ratio, showing that this possibility can be ruled out. Given the calibration of parameters in our experiment, i.e., for an intermediate endowment level, there exist asymmetric equilibria both in mixed and in pure strategies. Asymmetric mixed strategy equilibria are difficult to analyze, as we do not know how many they may be nor what they look like. However, we show that any mixed strategy equilibrium results in the same total expected bids (see Lemma 1). Thus, for any asymmetric equilibrium in mixed strategy, the aggregate prediction is the same as for the symmetric equilibrium.

Proposition 3 characterizes all the asymmetric equilibria in pure strategies, showing how, in some of them, the budget constraint gives rise to underdissipation of rents. For our parameter calibration, we show that in APA-RS there exists a unique pure strategy equilibrium such that two agents bid the entire endowment and the other two bid 0. Hence, total bids are the same as in the symmetric equilibrium, with an average expected bid of 400. In APAPG there exists a unique pure strategy equilibrium such that three agents bid the entire endowment and one agent bids 0. Noticeably, in this case total bids are lower than in the symmetric equilibrium, with an average of 600 per subject instead of of an expected individual bid of 640. Nevertheless, even taking into account this revenue loss in APA-PG, the model still predicts a positive interaction between APA and PG. Overall, we conclude that the negative interaction we observe in the experimental data cannot be explained by the existence of asymmetric equilibria. 


\section{Conclusions}

A wide body of experimental evidence provides support to the notion that agents' objective functions are not separable in economic incentives and social preferences. The empirical strategy generally followed in this literature is to compare the observed effects of a given incentive with the ones that would be predicted theoretically under separability. In this paper, we argued that the existing experimental studies based on this approach are generally not informative, as they only provide indirect evidence that is consistent with non-separability. In order to obtain causal evidence of non-separability, it is necessary to compare the effectiveness of economic incentives while also manipulating the elicitation of social preferences.

In order to illustrate this point, we presented an experiment based on a $2 \times 2$ design within a contest game, where we manipulate orthogonally the degree of competition of the incentive mechanism (lottery vs. all-pay auction) and the presence or absence of redistribution (public good vs. pure rent-seeking). Consistently with standard theory, we find that both stronger competition and the presence of the public good have a positive and significant effect on bidding. On the other hand, we find a negative interaction between stronger competition and the presence of the public good, leading us to reject the null hypothesis of separability between economic incentives and social preferences. This is an important finding, as it provides causal evidence that economic incentives may negatively affect pro-social behavior. More generally, our findings imply that social preferences may be relevant not only per se but also, and perhaps more importantly, for the optimal design of economic incentive mechanisms. 


\section{Appendix A - Theoretical predictions}

Note that expression (1) can be rearranged as

$$
\begin{aligned}
& \omega-\frac{n-\alpha}{n} x_{i}+\frac{\alpha}{n} \sum_{j \neq i} x_{j}+p\left(x_{i}, \mathbf{x}_{-i}\right) \Pi \\
& =\frac{\alpha}{n}\left(\omega+\sum_{j \neq i} x_{j}\right)+\frac{n-\alpha}{n}\left(\omega-x_{i}+p\left(x_{i}, \mathbf{x}_{-i}\right) \frac{n}{n-\alpha} \Pi\right) .
\end{aligned}
$$

From the above equation we conclude that the game we described is strategically equivalent to a standard all-pay contest in which $n$ agents endowed with a budget $\omega$ compete for a prize equal to $\frac{n}{n-\alpha} \Pi$. Thus, to simplify the analysis, we will analyze the latter.

\section{Lottery}

We begin by proving Proposition 1 .

Proof of Proposition 1. Notice first that in equilibrium total bids cannot be equal to zero. If this were the case any agent would have an incentive to bid $\varepsilon$ arbitrarily small and win the prize with certainty. Hence, $p^{L O T}\left(x_{i}, \mathbf{x}_{-i}\right)=$ $\frac{x_{i}}{\sum_{j \in N} x_{j}}$. We start by exploring symmetric equilibria. Let us rewrite $\omega-x_{i}+$ $\frac{x_{i}}{\sum_{j \in N} x_{j}} \frac{n}{n-\alpha} \Pi$ as

$$
\omega-x_{i}+\frac{x_{i}}{x_{i}+\sum_{j \neq i} x_{j}} \frac{n}{n-\alpha} \Pi .
$$

Differentiating the above expression with respect to $x_{i}$ and setting the derivative equal to zero we obtain the following first order condition

$$
\frac{\sum_{j \neq i} x_{j}}{\left(x_{i}+\sum_{j \neq i} x_{j}\right)^{2}} \frac{n}{n-\alpha} \Pi=1 .
$$

Solving with respect to $x_{i}$ we obtain player $i$ 's best response function

$$
x_{i}=-\sum_{j \neq i} x_{j}+\sqrt[2]{\frac{n}{n-\alpha} \Pi \sum_{j \neq i} x_{j}} .
$$

By symmetry, we can replace $x_{j}$ with $x_{i}$, obtaining

$$
x_{i}=-(n-1) x_{i}+\sqrt[2]{\frac{n}{n-\alpha} \Pi(n-1) x_{i}} .
$$


Solving for $x_{i}$ we obtain the following

$$
x_{i}^{*}=\frac{n-1}{n} \frac{\Pi}{n-\alpha} .
$$

When $\omega>\frac{n-1}{n} \frac{\Pi}{n-\alpha}$ the above expression represents the unique symmetric Nash equilibrium of LOT.

We continue the proof by showing that when $\omega \leq \frac{n-1}{n} \frac{\Pi}{n-\alpha}$ in equilibrium each player bids the whole endowment. Suppose that everyone but $i$ bids $\omega$. From (6) $i$ 's best response is equal to

$$
-(n-1) \omega+\sqrt[2]{\frac{n}{n-\alpha} \Pi(n-1) \omega} .
$$

Solving the following inequality $-(n-1) \omega+\sqrt[2]{\frac{n}{n-\alpha} \Pi(n-1) \omega}<\omega$ for $\omega$ we obtain

$$
\omega>\frac{n-1}{n} \frac{\Pi}{n-\alpha} .
$$

This implies that $i$ 's best response is less than $\omega$ only if $\omega>\frac{n-1}{n} \frac{\Pi}{n-\alpha}$, which contradicts our initial assumption. This proves that whenever budget constraints are binding it is an equilibrium to bid $\omega$.

Finally, from Theorem 1 in Cornes and Hartley (2005) we know that LOT has a unique Nash equilibrium in pure strategies. Hence, no other equilibria exist.

\section{All-pay auction}

In order to prove Proposition 2 we present the following two auxiliary lemmas. First of all, we demonstrate that there are no symmetric equilibria in pure strategies when $\omega>\frac{\Pi}{n-\alpha}$.

Lemma 1 If $\omega>\frac{\Pi}{n-\alpha}$ there are no symmetric equilibria in pure strategies.

Proof. Suppose, to the contrary, the existence of an equilibrium in which everyone bids $x$, receiving an expected payoff equal to $\frac{\Pi}{n-\alpha}-x$. Clearly $x \leq \frac{\Pi}{n-\alpha}$, otherwise an agent would rather bid zero. Any player would have an incentive to slightly raise her bid and win the prize, thus receiving a payoff equal to $\frac{n}{n-\alpha} \Pi-x-\varepsilon>\frac{\Pi}{n-\alpha}-x$, which contradicts the initial assumption.

The above lemma implies that, in order to characterize the symmetric equilibrium for the case $\omega>\frac{\Pi}{n-\alpha}$, we can simply focus on mixed strategies. Moreover the following key lemma states that, when $\omega>\frac{\Pi}{n-\alpha}$, any mixed strategy equilibrium results in full rent dissipation, i.e. total bids amount to $\frac{n}{n-\alpha} \Pi$. 
Lemma 2 If $\omega>\frac{\Pi}{n-\alpha}$, in any mixed strategy equilibrium total expected bids are equal to $\frac{n}{n-\alpha} \Pi$.

Proof. The proof consists of five parts.

i) In equilibrium no player can have a mass point at any bid $x \in(0, m)$, where $m=\min \left\{\omega, \frac{n}{n-\alpha} \Pi\right\}$. Suppose, to the contrary, that exactly one player has a mass point at $x \in(0, m)$. There is an interval $(x-\varepsilon, x)$, with $\varepsilon$ arbitrarily small, where no other agent puts positive density. If another player put density in that interval, moving all the density from $(x-\varepsilon, x)$ to $x$ would guarantee a discrete increase in the probability of winning at an infinitesimal cost. However, if no one else puts density on $(x-\varepsilon, x)$, then the bidder with mass at $x$ would have an incentive to move her mass lower. Now suppose that more than one player has a mass point at $x \in(0, m)$. Then there would be at least one player who could move her mass at $m+\varepsilon$, increasing her expected payoff.

ii) The lower bound of any player's randomization support must be zero. This follows directly from i). First of all, notice that all players must have the same lower bound in equilibrium. Suppose to the contrary that player $i$ 's lower bound is $l_{1}$ while $j$ 's lower bound is $l_{2}>l_{1}$. Clearly $i$ would not want to put any density on the interval $\left[l_{1}, l_{2}\right)$. Thus, it cannot be that different players have different lower bounds. Suppose now that the common lower bound is $l>0$. All players must have a mass point at $l$. If this was not the case those who do not have a mass point would be making a loss, but a player would rather bid zero than accepting a loss. However, we know from i) that there cannot be an atom at $l$.

iii) It cannot be that everyone has a mass point at zero. If everyone had an atom at zero, then any player would have an incentive to move that mass to a point $\varepsilon$ arbitrarily small.

iv) It cannot be that all but one player have a mass point at zero. Suppose to the contrary that player $i$ is the only one who does not place any mass at zero. Since, we know from ii) that the lower bound of $i$ 's randomization support must be zero, this implies that $i$ is receiving a strictly positive payoff while anybody else is making zero profit. If $\frac{n}{n-\alpha} \Pi<\omega$ it follows that the upper bound of $i$ 's support must be $u<\frac{n}{n-\alpha} \Pi$. Recall from i) that $i$ cannot put any mass at $u$, thus any other player has an incentive to deviate and bid $u$ with probability one. If $\frac{n}{n-\alpha} \Pi>\omega$ then $i$ 's upper bound can either be $u<\omega$ or coincide with $\omega$. In the first case we know there cannot be an atom at $u$ and thus, as before, anybody else would have an incentive to bid $u$. The same would happen if $\omega$ is $i$ 's upper bound but $i$ does not place any mass at that point: another player would have an incentive to bid $\omega$. The remaining possibility is that $i$ has a mass point at $\omega$. Recall however, that $i$ receives a positive payoff, while all other players are making zero profit. This implies that the others either do not put any mass at $\omega$ or place less mass than $i$. In the first case, a player would have an incentive to place mass at $\omega$, tying with $i$ and sharing her profit. In the second case, a player who is already tying 
with $i$ could profitably deviate by increasing the mass at $\omega$. This proves that it cannot be that all but one player have a mass point at zero.

v) At least two players do not place any mass at zero. This follows directly from iv) and implies that everyone's expected payoff must be zero. Hence, total expected bids are equal to $\frac{n}{n-\alpha} \Pi$.

We can now go on to prove Proposition 2.

Proof of Proposition 2. The proof is divided in three parts.

i) We start with the case $\omega \leq \frac{\Pi}{n-\alpha}$. It is easy to see that when all other players bid $\omega$ it is a best response to bid the whole endowment receiving a payoff equal to $\frac{\Pi}{n-\alpha}-\omega$. Clearly, no other symmetric equilibrium can exist, as an agent would have an incentive to outbid the others and win the prize with certainty, earning a higher payoff.

ii) We continue with the case $\frac{\Pi}{n-\alpha}<\omega<\frac{n}{n-\alpha} \Pi$. We know from Lemma 2 that rents are fully dissipated. It follows that the upper bound of the randomization support is equal to $\omega$ and that each agent must place a mass at $\omega$ such that they earn an expected payoff of zero. Hence, they must bid $\omega$ with a probability $q$ such that

$$
\frac{n}{n-\alpha} \Pi \sum_{j=0}^{n-1}\left(\begin{array}{c}
n-1 \\
j
\end{array}\right) \frac{q^{j}(1-q)^{n-1-j}}{j+1}-\omega=0
$$

The first term of equation (7) represents a player's expected prize when she bids $\omega$, given that everybody else bids $\omega$ with probability $q$. By applying the binomial theorem $\sum_{j=0}^{n-1}\left(\begin{array}{c}n-1 \\ j\end{array}\right) \frac{q^{j}(1-q)^{n-1-j}}{j+1}$ can be transformed into $\frac{1-(1-q)^{n}}{q n}$ and therefore equation (7) reduces to

$$
\frac{1-(1-q)^{n}}{q}=\frac{n-\alpha}{\Pi} \omega .
$$

Let us rewrite (8) in the following way

$$
q=\frac{\Pi}{(n-\alpha) \omega}\left[1-(1-q)^{n}\right] .
$$

Note that the RHS is equal to zero when $q=0$ and equal to $\frac{\Pi}{(n-\alpha) \omega}<1$ when $q=1$. Differentiating the RHS with respect to $q$ we obtain $\frac{\Pi}{(n-\alpha) \omega} n(1-q)^{n-1}$. This is positive for any $q$ and strictly greater than 1 when $q=0$. The second derivative is equal to $-\frac{\Pi}{(n-\alpha) \omega} n(n-1)(1-q)^{n-1}<0$. This implies that there is a unique $q^{*} \in(0,1)$ that solves equation $(8)$.

Since players have a mass point at $\omega$ there exists $\bar{x}<\omega$ such that no one puts any density in the interval $(\bar{x}, \omega)$. Suppose the contrary. Then moving all this density to $\omega$ would produce a discrete increase in the probability of winning at a cost sufficiently low to make it worth deviating. The value of $\bar{x}$ must be such that an agent bidding $\bar{x}$ earns zero expected profit. Since each player bids more than $\bar{x}$ with probability $q$ it must be $\bar{x}=(1-q)^{n-1} \frac{n}{n-\alpha} \Pi$. 
We know from point i) of Lemma 2 that players cannot have a mass point at any bid $x \in(0, \bar{x})$. Moreover, we know from point ii) of Lemma 2 that the lower bound is equal to zero, and from point iii) of Lemma 2 that they cannot all have a mass point at zero. Finally, since there are no mass points there cannot be a gap in the support between 0 and $\bar{x}$. Suppose there was an interval $(a, b)$ in which no one puts any density. By bidding $x \in(a, b)$ an agent would have the same probability of winning guaranteed by $b$ at a strictly lower cost. From this follows that, in a symmetric equilibrium, players randomize on the support $[0, \bar{x}]$ according to a distribution $F(x)$ such that

$$
\frac{n}{n-\alpha} \Pi F(x)^{n-1}-x=0 .
$$

The unique solution to the above equation is $F(x)=\left(\frac{n-\alpha}{n} \frac{x}{\Pi}\right)^{\frac{1}{n-1}}$, thus representing the unique symmetric Nash equilibrium.

iii) We conclude with the case $\omega \geq \frac{n}{n-\alpha} \Pi$. First, we know from Lemma 2 that rents are fully dissipated. It follows that the upper bound of the randomization support is equal to $\frac{n}{n-\alpha} \Pi$. Moreover, in a symmetric equilibrium agents cannot have an atom at the upper bound, otherwise they would incur a loss. Following the proof of the previous point, in the unique symmetric Nash equilibrium players randomize on the support $\left[0, \frac{n}{n-\alpha} \Pi\right]$ according to $F(x)=\left(\frac{n-\alpha}{n} \frac{x}{\Pi}\right)^{\frac{1}{n-1}}$.

\section{All-pay auction's asymmetric equilibria}

We know from Proposition 2 that there exists a unique symmetric equilibrium for any value of $\omega$. If $\omega \leq \frac{\Pi}{n-\alpha}$ the symmetric equilibrium is such that everyone bids the whole endowment. Clearly, when $\omega<\frac{\Pi}{n-\alpha}$ rents are not fully dissipated, with total bids equal to $n \omega<\frac{n}{n-\alpha} \Pi$. However, in the symmetric equilibrium full dissipation occurs whenever $\omega \geq \frac{\Pi}{n-\alpha}$. Moreover, we know that if $\omega>\frac{\Pi}{n-\alpha}$ : a) no symmetric pure strategy equilibria exist (Lemma 1) and b) rents are fully dissipated in any mixed strategy equilibrium, be it symmetric or asymmetric (Lemma 2). Hence, the question that arises is i) whether there exist asymmetric pure strategy equilibria associated with less than full dissipation when $\omega \geq \frac{\Pi}{n-\alpha}$; and ii) whether there exist asymmetric equilibria producing a revenue lower than $n \omega$ when $\omega<\frac{\Pi}{n-\alpha}$. In the next proposition we characterize all pure strategy asymmetric equilibria and the corresponding total bids.

\section{Proposition 3}

If $\omega<\frac{\Pi}{n-\alpha}$ there exist no asymmetric equilibria.

If $\frac{1}{k+1} \frac{n}{n-\alpha} \Pi<\omega<\frac{1}{k} \frac{n}{n-\alpha} \Pi$, with $k=1, \ldots, n-1$, there exists a unique pure strategy equilibrium such that $k$ players bid $\omega$ and $n-k$ players bid zero. Total bids are equal to $k \omega<\frac{n}{n-\alpha} \Pi$. 
If $\omega=\frac{1}{k+1} \frac{n}{n-\alpha} \Pi$, with $k=2, \ldots, n-1$, there exist only two pure strategy equilibria. In one $k$ players bid $\omega$ and $n-k$ bid zero, with total bids equal to $k \omega<\frac{n}{n-\alpha} \Pi$. In the other equilibrium $k+1$ agents bid $\omega$ and $n-k-1$ bid zero, with total bids equal to $\frac{n}{n-\alpha} \Pi$.

If $\omega=\frac{1}{2} \frac{n}{n-\alpha} \Pi$, there exists a unique pure strategy equilibrium such that 2 agents bid $\omega$ and $n-2$ bid zero. Total bids are equal to $\frac{n}{n-\alpha} \Pi$.

If $\omega \geq \frac{n}{n-\alpha} \Pi$ there exist no asymmetric pure strategy equilibria.

Proof. The proof is divided in five parts, one for each of the above statements.

i) We know from Proposition 2 that there exists a symmetric equilibrium in which every agent bids $\omega$. Suppose, contrary to the statement, the existence of an asymmetric equilibrium characterized by the following strategy profile $\left\{x_{1}, \ldots, x_{i}, \ldots x_{n}\right\}$. Call $x_{h}$ the highest bid. If $x_{h}<\omega$ any player would have an incentive to bid $x_{h}+\varepsilon$ and win the prize for sure. If $x_{h}=\omega$ whoever is bidding less than $\omega$ must be bidding zero, as any other bid would result in a negative payoff. However, a bid equal to $\omega$ would guarantee this agent a profit at least equal to $\frac{n}{n-1} \frac{\Pi}{n-\alpha}-\omega>0$. It follows that no asymmetric equilibria exist.

ii) Let us start by proving that this is indeed an equilibrium. On the one hand, none of the agents who bid zero has an incentive to make any bid $x \in(0, \omega)$ as this would result in a negative payoff. Moreover, none of them has an incentive to bid $\omega$ either, as this would guarantee a payoff equal to $\frac{1}{k+1} \frac{n}{n-\alpha} \Pi-\omega<0$. On the other hand, each of the $k$ players who bid $\omega$ earns a payoff equal to $\frac{1}{k} \frac{n}{n-\alpha} \Pi-\omega>0$ and therefore has no incentive to drop her bid. Note that, since $\omega<\frac{1}{k} \frac{n}{n-\alpha} \Pi$, revenue is strictly less than $\frac{n}{n-\alpha} \Pi$, i.e., rents are not fully dissipated. We now prove uniqueness among pure strategy equilibria. First, recall from Lemma 1 that no symmetric pure strategy equilibria exist when $\omega>\frac{\Pi}{n-\alpha}$. Hence, we need to rule out asymmetric pure strategy equilibria. Clearly, there cannot exist an equilibrium in which no one bids $\omega$, as any agent would have an incentive to outbid all the others. Moreover, if at least one agent bids $\omega$ then no one else would want to bid $x \in(0, \omega)$. Finally, if $l<k$ players were bidding $\omega$, then at least one of the remaining agents would have an incentive to also bid $\omega$, thus earning $\frac{1}{l+1} \frac{n}{n-\alpha} \Pi-\omega>0$. Hence no other asymmetric pure strategy equilibria exist.

iii) Consider the case in which $k$ players bid $\omega$ and $n-k$ bid zero. Each of the $k$ players earns $\frac{1}{k} \frac{n}{n-\alpha} \Pi-\omega>0$ and thus has no incentive to deviate; each of the remaining players is indifferent between not bidding and bidding $\omega$. Since $\omega<\frac{1}{k} \frac{n}{n-\alpha} \Pi$, revenue is strictly less than $\frac{n}{n-\alpha} \Pi$, i.e., rents are not fully dissipated. When $k+1$ players bid $\omega$ each one of them is indifferent between bidding the whole budget or bidding zero; clearly, the remaining $n-k-1$ players have no incentive to bid more than zero. In this equilibrium 
rents are fully dissipated. The proof of uniqueness outlined in ii) rules out the existence of other pure strategy equilibira in this case too.

iv) This is equivalent to the second equilibrium outlined in iii), i.e., $k+1$ players bid $\omega$ and the others abstain. Each of the two players who bid $\omega$ is indifferent between bidding the whole endowment and not bidding; the remaining players have no incentive to make a positive bid, and rents are fully disipated. Differently from iii), $k$ players bidding $\omega$ and $n-k$ bidding zero is not an equilibrium. This is because, in this case, $k=1$ : the active player would not be tying with anyone else and would therefore have an incentive to bid $\varepsilon$. Following the proof of ii) we can rule out any other equilibrium in pure strategies.

iv) No more than one player can bid $\frac{n}{n-\alpha} \Pi$ in equilibrium, as this would result in a negative payoff. If exactly one player bids $\frac{n}{n-\alpha} \Pi$ it is a best response for the others to bid zero; however, the agent who is bidding $\frac{n}{n-\alpha} \Pi$ has an incentive to drop her bid to $\varepsilon$ arbitrarily close to zero. Finally if the highest bid is $h<\frac{n}{n-\alpha} \Pi$ anyone bidding less than $h$ has an incentive to bid $h+\varepsilon$. This proves that no asymmetric pure strategy equilibria exist.

The above proposition shows that if $\omega<\frac{\Pi}{n-\alpha}$ the symmetric equilibrium outlined in Proposition 2 is also the unique equilibrium. Asymmetric pure strategy equilibria only exist in the range $\omega \in\left[\frac{\Pi}{n-\alpha}, \frac{n}{n-\alpha} \Pi\right)$. If $\frac{1}{k+1} \frac{n}{n-\alpha} \Pi<$ $\omega<\frac{1}{k} \frac{n}{n-\alpha} \Pi$, for $k=1, \ldots, n-1$, the pure strategy equilibrium is unique, with total bids equal to with $k \omega<\frac{n}{n-\alpha} \Pi$. If $\omega=\frac{1}{k+1} \frac{n}{n-\alpha} \Pi$, for $k=2, \ldots, n-1$, there exist two pure strategy equilibria with total bids equal to either $k \omega<$ $\frac{n}{n-\alpha} \Pi$ or $(k+1) \omega=\frac{n}{n-\alpha} \Pi .^{13}$ Finally, when $\omega=\frac{1}{2} \frac{n}{n-\alpha} \Pi$ there exists a unique equilibrium in which 2 agents bid $\omega$ while the others bid zero, total bids being equal to $\frac{n}{n-\alpha} \Pi$. Hence, when $\omega \in\left[\frac{\Pi}{n-\alpha}, \frac{n}{n-\alpha} \Pi\right)$ there exists one equilibrium associated with under-dissipation of rents.

Let us conclude by applying the above proposition to the specific parametrization used in our experiment. Recall that $n=4, \omega=800$ and $\Pi=1600$, while either $\alpha=0$ or $\alpha=1.5$. When $\alpha=0$, we are in the case $\omega=\frac{1}{2} \frac{n}{n-\alpha} \Pi$ and thus we have a unique pure strategy equilibrium such that two agents bid 800 while the other two bid zero. This equilibrium results in full rent dissipation, as in any mixed strategy equilibrium. When $\alpha=1.5$ we fall in the case $\frac{1}{k+1} \frac{n}{n-\alpha} \Pi<\omega<\frac{1}{k} \frac{n}{n-\alpha} \Pi$, with $k=3$. Hence, there exists a unique pure strategy equilibrium producing under-dissipation. Three players bid 800 and one bids zero, with total bids equal to 2400 .

\footnotetext{
${ }^{13}$ Note that all of these equilibria are asymmetric, with the exception of the case $\omega=$ $\frac{\Pi}{n-\alpha}$. In this case one of the two pure strategy equilibria coincides with the symmetric equilibrium in which everyone bids $\omega$.
} 


\section{Appendix B - Instructions}

[Instructions for Session 1]

Welcome. Thanks for participating in this experiment. If you follow the instructions carefully and make good decisions you can earn an amount of money that will be paid to you in cash at the end of the experiment. During the experiment you are not allowed to talk or communicate in any way with other participants. If you have any questions raise your hand and one of the assistants will come to you to answer it. The rules that you are reading are the same for all participants.

\section{General Rules}

- There are 24 subjects participating in this experiment.

- The experiment will consist of 40 rounds.

- In every round you will be randomly and anonymously assigned to a group of 4 subjects and you will be able to earn an amount of euros based on your and others' choices.

- In every round each of the 4 participants will be assigned an endowment of 8 euros and will be able to win a prize of 16 euros by buying tickets (between a minimum of 0 and a maximum of 800) at the cost of 1 euro cent per ticket.

- At the end of the experiment the computer will randomly select one of the 40 rounds.

- You will be paid the amount of euros you won in the selected round in cash.

\section{How your earnings are determined, rounds 1 - 10}

- The computer randomly selects the winning ticket among all the tickets purchased by the members of your group. The owner of the winning ticket wins the prize.

- Thus your probability of winning the prize is given by the number of tickets you bought divided by the total number of tickets bought by members of your group.

- In case no tickets are purchased, no one wins the price.

Example: if you buy 300 tickets and each one of the other subjects buys 200 tickets, you earn $8-3=5$ euros which you keep for yourself and you have a probability equal to $\frac{300}{900}=\frac{1}{3}$ of winning the prize. 
[Control questions] Calling A,B,C,D the four members of your group and assuming that in a given round the tickets bought by A,B,C,D are, respectively, 200, 400, 800 and 600:

- How many euros does A keep for herself?

- What is the probability that A wins the prize?

- How many euros does B keep for herself?

- What is the probability that $\mathrm{B}$ wins the prize?

\section{How your earnings are determined, rounds $11-20$}

- The computer randomly selects the winning ticket among all the tickets purchased by the members of your group. The owner of the winning ticket wins the prize.

- Thus your probability of winning the prize is given by the number of tickets you bought divided by the total number of tickets bought by members of your group.

- In case no tickets are purchased, no one wins the price.

Example: if you buy 300 tickets and each one of the other subjects buys 200 tickets, you earn $8-3=5$ euros which you keep for yourself and you have a probability equal to $\frac{300}{900}=\frac{1}{3}$ of winning the prize.

- Moreover, the total amount spent by members of your group to buy tickets is multiplied by 1.5 and divided equally among all group members.

[Control questions] Calling A,B,C,D the four members of your group and assuming that in a given round the tickets bought by A,B,C,D are, respectively, 200, 400, 800 and 600:

- How many euros does A keep for herself?

- How many euros will A obtain from the distribution of the total amount spent by members of your group for buying tickets?

- What is the probability that A wins the prize?

- How many euros does B keep for herself?

- How many euros will B obtain from the distribution of the total amount spent by members of your group for buying tickets?

- What is the probability that $B$ wins the prize? 


\section{How your earnings are determined, rounds 21 - 30}

- The member of your group who buys the highest number of tickets wins the prize.

- If two or more group members buy the same number of tickets, and that is also the highest number, the prize is randomly assigned among those participants.

Example: if you buy 300 tickets, and each of the other group members buys 200 tickets, you win the prize.

Example: if you buy 300 tickets, and each of the other group members buys 400 tickets, you don't win the prize.

Example: if you and another group member buy 300 tickets, while the other two buy 200 tickets each, you win the prize with probability $\frac{1}{2}$.

[Control questions] Calling A,B,C,D the four members of your group and assuming that in a given round the tickets bought by A,B,C,D are, respectively, 200, 400, 800 and 600:

- How many euros will be earned by A?

- Who will win the prize?

Assuming that in a given round the tickets bought by A,B,C,D are, respectively, 200, 400, 600 and 600:

- How many euros will be earned by B?

- What is the probability that $\mathrm{B}$ wins the prize?

- What is the probability that $\mathrm{C}$ wins the prize?

\section{How your earnings are determined, rounds $31-40$}

- The member of your group who buys the highest number of tickets wins the prize.

- If two or more group members buy the same number of tickets, and that is also the highest number, the prize is randomly assigned among those participants.

Example: if you buy 300 tickets, and each of the other group members buys 200 tickets, you win the prize.

Example: if you buy 300 tickets, and each of the other group members buys 400 tickets, you don't win the prize.

Example: if you and another group member buy 300 tickets, while the other two buy 200 tickets each, you win the prize with probability $\frac{1}{2}$. 
- Moreover, the total amount spent by members of your group to buy tickets is multiplied by 1.5 and divided equally among all group members.

[Control questions] Calling A,B,C,D the four members of your group and assuming that in a given round the tickets bought by A,B,C,D are, respectively, 200, 400, 800 and 600:

- How many euros will be earned in total by A?

- How many euros will A obtain from the distribution of the total amount spent by members of your group for buying tickets?

- Who will win the prize?

Assuming that in a given round the tickets bought by A,B,C,D are, respetively, 300, 500, 600 and 600 :

- How many euros will be earned by B?

- How many euros will B obtain from the distribution of the total amount spent by members of your group for buying tickets?

- What is the probability that B wins the prize?

- What is the probability that $\mathrm{C}$ wins the prize? 


\section{References}

Baye, M., Kovenock, D. and C. de Vries (1996). "The all-pay auction with complete information", Economic Theory, 8, 291-305.

Benabou, R. and J. Tirole (2006). "Incentives and prosocial behavior", American Economic Review, 96(5), 1652-1678.

Bowles, S. and S. Polanía-Reyes (2012). "Economic Incentives and Social Preferences: Substitutes or Complements?", Journal of Economic Literature, 50(2), 368-425.

Carpenter, J., J. Holmes and P.H. Matthews (2008). "Charity auctions: A field experiment", The Economic Journal, 118, 92-113.

Corazzini, L., Faravelli, M. and L. Stanca (2010). "A prize to give for: An experiment on public good funding mechanisms", The Economic Journal, 120(547), 944-967.

Cornes, R. and R. Hartley (2005). "Asymmetric contests with general technologies", Economic Theory, 26, 923-946.

Davis, D.D., L. Razzolini, R.J. Reilly and B.J. Wilson (2006). "Raising revenues for charity: Auctions versus lotteries", in: D.D. Davis and R.M. Isaac (eds.), Research in Experimental Economics Vol. 11, JAI Press, Greenwich, 47-91.

Davis, D. and R. Reilly (1998). "Do too many cooks always spoil the stew? An experimental analysis of rent-seeking and the role of a strategic buyer", Public Choice, 95(1), 89-115.

Dechenaux, E., Kovenock, D. and R. M. Sheremeta (2014). "A survey of experimental research on contests, all-pay auctions and tournaments", Experimental Economics, forthcoming.

Duffy, J. and A. Matros (2012). "All-Pay Auctions vs. Lotteries as Provisional Fixed-Prize Fundraising Mechanisms: Theory and Evidence", Working Papers 448, University of Pittsburgh, Department of Economics.

Eisenkopf, G., S. Teyssier, (2013). "Envy and loss aversion in tournaments", Journal of Economic Psychology, 34, 240ï $\frac{1}{2} 255$.

Faravelli, M. and L. Stanca (2012). "Single versus multiple-prize all-pay auctions to finance public goods: An experimental analysis", Journal of Economic Behavior and Organization, 81(2), 677-688.

Fehr, E. and K. Shmidt (1999). "A theory of fairness, competition and cooperation", Quarterly Journal of Economics, 114(3), 817-868. 
Fischbacher, U. (2007). "z-Tree: Zurich Toolbox for Ready-made Economic Experiments", Experimental Economics, 10, 171-178.

Gneezy, U., Meier, S. and P. Rey-Biel (2011). "When and Why Incentives (Don't) Work to Modify Behavior" Journal of Economic Perspectives, $25(4), 191-210$.

Goeree, J. K., Maasland, E., Onderstal, S. and J. Turner (2005). "How (Not) to Raise Money", Journal of Political Economy, 113(4), 897-926.

Herrmann, B. and H. Orzen (2008). "The appearance of homo rivalis: Social preferences and the nature of rent seeking", Discussion Papers 2008-10, The Centre for Decision Research and Experimental Economics, School of Economics, University of Nottingham.

Konrad, K. (2009). Strategy and Dynamics in Contests, Oxford University Press.

Kreps, D. (1997). "Intrinsic Motivation and Extrinsic Incentives", American Economic Review, 87(2), 359-364.

Landry, C., Lange, A., List, J.A., Price, M.K. and N. Rupp (2006). "Toward an Understanding of the Economics of Charity: Evidence from a Field Experiment", Quarterly Journal of Economics, 121(2), 747-782.

Lange, A., List, J. A. and M. K. Price (2007). "Using Lotteries to Finance Public Goods: Theory and Experimental Evidence", International Economic Review, 48, 901-927.

Levitt, S. and J. List (2007). "What Do Laboratory Experiments Measuring Social Preferences Reveal About the Real World?", Journal of Economic Perspectives, 21(2), 153-174.

Morgan, J. (2000). "Financing Public Goods by Means of Lotteries", Review of Economic Studies, 67, 761-784.

Morgan, J. and M. Sefton, M. (2000). "Funding Public Goods with Lotteries: Experimental Evidence", Review of Economic Studies, 67, 785-810.

Onderstal, S., Schram, A. and A. Soetevent (2013). "Bidding to give in the field", Journal of Public Economics, 105, 72-85.

Orzen, H. (2008). "Fundraising through Competition: Evidence from the Lab", Discussion Papers 2008-11, The Centre for Decision Research and Experimental Economics, School of Economics, University of Nottingham. 
Potters, J., de Vries, C. and F. van Winden (1998). "An experimental investigation of rational rent seeking", European Journal of Political Economy, 14(4), 783-800.

Price, C. R. and R. M. Sheremeta (2011). "Endowment effects in contests", Economics Letters, 111, 217-219.

Rabin, M. (1993). "Incorporating fairness into game theory and economics", American Economic Review, 83, 1281-1302.

Savikhin, A. C. and R. M. Sheremeta (2011). "Simultaneous decisionmaking in competitive and cooperative games", Economic Inquiry, 51, 1311-1323.

Sheremeta, R. M. (2010). "Experimental Comparison of Multi-stage and One-stage Contests", Games and Economic Behavior, 68, 731-747.

Sheremeta, R. M., Masters, W. and T. Cason (2012). "Winner-Take-All and Proportional-Prize Contests: Theory and Experimental Results, Working Papers 12-04, Chapman University, Economic Science Institute.

Sheremeta, R. M. (2013). "Overbidding and Heterogeneous Behavior in Contest Experiments", Journal of Economic Surveys, 27, 491-514.

Schram, A. and S. Onderstal (2009). "Bidding to Give: An Experimental Comparison of Auctions for Charity", International Economic Review, 50(2), 431-457.

Schmidt, K. (2011). "Social Preferences and Competition", Journal of Money, Credit and Banking, 43, 207-231.

Tullock, G. (1980) "Efficient Rent-Seeking", In J. Buchanan et al., eds., Towards a theory of the rent-seeking society, College Station, Tx: Texas A\&M University Press. 